\title{
Caroli Disease
}

National Cancer Institute

\section{Source}

National Cancer Institute. Caroli Disease. NCI Thesaurus. Code C84619.

A rare congenital disorder characterized by cystic dilatation of the intrahepatic bile ducts.

It is associated with cholangitis and the formation of stones. 